\title{
Sozialindikative Planung der regionalen ärztlichen Versorgung
}

\author{
Ein Diskussionsbeitrag für Berlin am Beispiel \\ der Psychotherapeuten und Hausärzte
}

\section{MARIO CZAJA, GERHARD MEINLSCHMIDT, SUSANNE BETTGE}

Mario Czaja (CDU) ist Senator für Gesundheit und Soziales in Berlin

Prof. Dr. Gerhard Meinlschmidt ist Referatsleiter in der Senatsverwaltung für Gesundheit und Soziales Berlin und lehrt an der Berlin School of Public Health der Charité - Universitätsmedizin

Dr. Susanne Bettge ist Mitarbeiterin in der Senatsverwaltung für Gesundheit und Soziales Berlin

\author{
Das Versorgungsstrukturgesetz eröffnet neue \\ Spielräume für eine flexiblere ambulante \\ ärztliche Bedarfsplanung und gibt den Ländern \\ erweiterte Mitsprachemöglichkeiten im Hinblick \\ auf die Bedarfsplanung und sektorübergreifende \\ Fragestellungen. Diesen Gestaltungsspielraum will das \\ Land Berlin in partnerschaftlicher Zusammenarbeit \\ mit den Akteuren der Selbstverwaltung nutzen. \\ Als Diskussionsbeitrag zur Weiterentwicklung \\ der Bedarfsplanung wird exemplarisch für Berlin \\ die regionale ärztliche Versorgung analysiert.
}

\section{Einleitung und Hintergrund}

Die Sicherstellung einer medizinisch angemessenen, wohnortnahen, für alle gleichermaßen zugänglichen ärztlichen Versorgung wird als Aufgabe der öffentlichen Daseinsvorsorge betrachtet und unterliegt als solche in hohem Maße staatlicher Regulation (Kopetsch, 2011). Im Zuge der Reformen im Gesundheitswesen durch das Versorgungsstrukturgesetz hat das Thema der angemessenen ärztlichen Versorgung wieder an Aktualität gewonnen. Vor dem Hintergrund des demografischen Wandels mit einem steigenden Anteil älterer und hochbetagter Menschen an der Gesamtbevölkerung sowie einem anhaltenden Fortzug insbesondere jüngerer Menschen aus ländlichen Regionen in Verbindung mit dem stetigen medizinisch-technischen Fortschritt werden Befürchtungen geäußert, die derzeitige Versorgung könnte zukünftig nicht mehr ausreichend sein.

In Deutschland finden die Planung und Regulation ärztlicher Versorgung getrennt für den stationären und ambulanten Sektor statt, eine sektorübergreifende Versorgungsplanung existiert bisher nicht. Der Sicherstellungsauftrag für die stationäre Versorgung obliegt den Ländern, die Sicherstellung der ambulanten ärztlichen Versorgung wurde dagegen den Kassenärztlichen Vereinigungen übertragen (SGB V, $\mathbb{S} \mathbb{S} 72-76$ ). Die hier vorgestellten Analysen und Überlegungen beziehen sich ausschließlich auf die ambulante vertragsärztliche Versorgung.

Die Zulassung niedergelassener Vertragsärzte erfolgt nach den Maßgaben der Bedarfsplanungsrichtlinie. In ihrer heutigen Form basiert die Bedarfsplanungsrichtlinie auf dem Gesundheitsstrukturgesetz von 1993. Für 14 Arztgruppen sind Allgemeine Verhältniszahlen (AVZ) festgelegt, die bestimmen, auf wie viele Einwohner jeweils ein Arzt der entsprechenden Arztgruppe kommen soll. Dafür werden die Gemeinden in Abhängigkeit von ihrer Zentralität und Einwohnerdichte bundesweit in zehn Regionalklassen eingeteilt, für die jeweils unterschiedliche EinwohnerArzt-Relationen (AVZ) pro Arztgruppe festgelegt wurden. In Ermangelung unanfechtbarer Kriterien zur Feststellung des Versorgungsbedarfs und eines 
absoluten Maßstabs für eine bedarfsgerechte Versorgung wurde dabei auf Erfahrungswerte aus der Vergangenheit zurückgegriffen. Dabei wurden die tatsächlichen Ärzte- und Einwohnerzahlen zu bestimmten Stichtagen herangezogen, um deren Durchschnitt als AVZ für die entsprechende Regionalklasse festzulegen.

Der Versorgungsgrad für jede Arztgruppe errechnet sich, indem die tatsächliche Zahl der Ärzte pro Einwohner ins Verhältnis zur AVZ gesetzt wird. Wird ein Versorgungsgrad von $110 \%$ überschritten, so wird dies als Überversorgung definiert und der Planungsbereich wird für diese Arztgruppe für Neuzulassungen gesperrt. Eine Unterschreitung eines Versorgungsgrades von $75 \%$ in der hausärztlichen Versorgung bzw. von $50 \%$ in der fachärztlichen Versorgung wird als Unterversorgung definiert.

Inzwischen wird die Bedarfsplanung von verschiedenen Seiten als zu starr kritisiert und für überarbeitungsbedürftig gehalten. Die von der Bedarfsplanungsrichtlinie ausgehenden Steuerungsmöglichkeiten sind auf das Sperren von Planungsregionen für neue Niederlassungen ambulanter Ärzte beschränkt. Dennoch ist die Zahl niedergelassener Ärzte bundesweit seit ihrem Inkrafttreten stetig weiter angestiegen (Klose \& Rehbein 2011). Eine Steuerung des Versorgungsangebots (Uhlemann \& Lehmann 2011) und insbesondere eine Verlagerung von Arztsitzen aus überversorgten in unterversorgte Regionen finden kaum statt.

\section{Die bestehende Bedarfsplanung ist zu starr und überarbeitungs- bedürftig.}

Die Kritik an der Bedarfsplanung in der jetzigen Form zielt nicht nur auf die mangelnden Steuerungsmöglichkeiten der ambulanten Versorgung, sondern auch auf die mangelnde fachlich-theoretische oder wissenschaftlich-empirische Begründung der Allgemeinen Verhältniszahlen. Statt die zum jeweiligen Stichtag vorgefundenen Arztzahlen zum Maßstab bedarfsgerechter Versorgung zu erheben, wird eine morbiditätsorientierte Bedarfsplanung gefordert (Mühl- bacher \& Wessels 2011). Jedoch kann die ärztlich diagnostizierte Krankheitslast nicht allein als Maß für den Versorgungsbedarf herangezogen werden, weil auch die Nachfrage nach ärztlichen Versorgungsleistungen in gewissem Ausmaß angebotsgesteuert ist (Mühlbacher \& Wessels 2011). Daher wird die zusätzliche Einbeziehung von soziodemografischen und Bevölkerungsprognosedaten (Kopetsch 2011) sowie subjektiven Gesundheitseinschätzungen (Mühlbacher \& Wessels 2011) in die Bedarfsplanung gefordert.

Eine erste Anpassung der Bedarfsplanungsrichtlinie an die Bevölkerungsentwicklung wurde im November 2010 mit der Einführung eines Demografiefaktors vorgenommen, der der überproportionalen Inanspruchnahme des Gesundheitswesens durch ältere Bevölkerungsgruppen Rechnung tragen soll. Mit dem am 1.1.2012 in Kraft getretenen Versorgungsstrukturgesetz wird die Bedarfsplanung flexibilisiert, und den Ländern werden bisher nicht vorhandene Gestaltungsmöglichkeiten eingeräumt. Die konkrete Nutzung dieser Spielräume durch den Gemeinsamen Bundesausschuss G-BA und die Landesausschüsse sowie die möglichen Auswirkungen bleiben abzuwarten.

Mit der vorliegenden Untersuchung der Versorgungssituation in Berlin wird ein Diskussionsbeitrag zur Frage der bedarfsgerechten ambulanten Versorgung geleistet, der einen Ausgangspunkt für weiterführende Überlegungen zur bedarfsgerechten Versorgung und ihrer Steuerung darstellen kann. Am Beispiel der Arztgruppe der Psychotherapeuten mit einer bundesweit, aber auch innerhalb Berlins feststellbaren hohen Inhomogenität der Verteilung der Arzt- bzw. Therapeutensitze und der Arztgruppe

der Hausärzte mit einer vergleichsweise homogenen Ärzteverteilung wird ein Modell zur Adjustierung der Arztzahlen anhand der Sozialstruktur und der Morbidität der Bevölkerung entwickelt. Dabei wurden diese beiden Arztgruppen mit ihrer recht unterschiedlichen Versorgungsform exemplarisch ausgewählt, um die Modellwirkungen kontrastierend zu vergleichen. Eine Übertragbarkeit der Ergebnisse auf weitere Arztgruppen ist nur bedingt möglich und sollte in Über- legungen zur Weiterentwicklung des Modells einbezogen werden.

Mit dem Modell wird nicht der Versuch unternommen, die vorhandenen Versorgungsstrukturen zu erklären, sondern es wurde auf der Grundlage inhaltlich-theoretischer Überlegungen zu Parametern eines bedarfsgerechten Versorgungsangebots spezifiziert.

Das Modell basiert auf den tatsächlichen Arztzahlen in Berlin im Jahr 2010 und klammert damit die Frage aus, ob die aktuelle Versorgung dem Bedarf entspricht oder ob eine Über- oder Unterversorgung vorliegt. Der im Jahr 2010 in die Bedarfsplanung aufgenommene Demografiefaktor, der durch die Kassenärztlichen Vereinigungen jeweils in ihrem Planungsbereich zum Ansatz gebracht wird, ist nicht Gegenstand der Modellbildung. Auswertungen zur Morbidität der Bevölkerung basieren auf den Abrechnungsdaten der KV Berlin des Jahres 2007. Zwischenzeitlich erfolgte Veränderungen oder Anpassungen konnten entsprechend nicht berücksichtigt werden.

Mit den Modellkomponenten Sozialstruktur und Morbidität wird eine sozialräumlich differenzierte Umverteilung der Arztsitze vorgenommen. Es wurde somit ein relativer Ansatz gewählt, der lediglich die Allokation bereits vorhandener Versorgungskapazitäten thematisiert und keine Aussagen über ein angemessenes, kostengünstiges und bedarfsgerechtes Versorgungsangebot in seiner absoluten Höhe trifft.

\section{Methodik}

Die Modellbildung erfolgt - wie bereits eingangs erwähnt - für die Arztgruppen Psychotherapeuten und Hausärzte, um vergleichend einander gegenüberstellen zu können.

Die Arztgruppe der Psychotherapeuten stellt im Rahmen der ambulanten Versorgung in mehrerer Hinsicht einen Spezialfall dar. Zunächst einmal setzt sie sich zusammen aus ärztlichen und nichtärztlichen Psychotherapeuten, wobei letztere sich noch weiter aufgliedern in Psychologische Psychotherapeuten und Kinder- und Jugendlichenpsychotherapeuten. Für die drei Untergruppen gelten in der Bedarfsplanungsrichtlinie spezielle Quotenregelungen innerhalb der Arztgruppe der Psychotherapeuten, so dass eine Zulassung in einer Unter- 
gruppe, die in der Planungsregion ihre Quote noch nicht erreicht hat, auch bei Sperrung der Region aufgrund von Überversorgung möglich ist.

Zudem bieten Psychotherapeuten eine von den anderen Arztgruppen, insbesondere von der primärärztlichen Versorgung, deutlich verschiedene Versorgungsform an. Die Patientinnen und Patienten werden nicht in offenen Sprechstunden versorgt, sondern nach Voranmeldung in einem intensiven, längerfristig angelegten Therapieprozess diagnostiziert und behandelt. Deshalb verzeichnet die Gruppe der Psychotherapeuten pro Abrechnungszeitraum eine erheblich geringere Anzahl von Patientinnen und Patienten bzw. Abrechnungsscheinen als alle übrigen Arztgruppen.

\section{Psychotherapeuten stellen im Rahmen der ambulanten Versorgung einen Spezialfall dar.}

Die Gruppe der Hausärzte dagegen setzt sich zusammen aus Allgemeinärzten und hausärztlich tätigen Internisten, die eine primärärztliche Versorgung anbieten, die in der ausschließlichen Behandlung von Patientinnen und $\mathrm{Pa}-$ tienten bestehen kann, sich aber auch auf die Mitbehandlung in Kooperation mit anderen Arztgruppen oder aber die Erstbehandlung mit anschließender Überweisung an eine Ärztin oder einen Arzt einer bestimmten Fachgruppe erstrecken kann.

Als Analyseebene werden die 12 Berliner Bezirke ausgewählt. Damit findet eine Differenzierung unterhalb der derzeitigen einheitlichen Planungsregion statt. Anhand des regionalen Bezugssystems für Berlin ließe sich die Analyse auch noch weit detaillierter durchführen. Jedoch wird für einen ersten Analyseansatz eine nicht zu kleinräumige Betrachtung für sinnvoll gehalten.

\section{Modellparameter}

In die Modellbildung gehen als Parameter die tatsächlichen Einwohner- und Ärztezahlen ein. Die Sozialstruktur wird mit dem Sozialindex I des Berliner Sozialstrukturatlasses 2008 (Meinlschmidt 2009) erfasst, die Morbiditätsstruktur mit dem durch Analyse der Daten der
KV Berlin 2007 gewonnenen Morbiditätsindex I (Meinlschmidt 2011). Ergänzend werden bezirkliche und überbezirkliche Versorgungsstrukturen sowie Indikatoren der ärztlichen Auslastung betrachtet und mit den Modellparametern in Beziehung gesetzt. Die genannten Parameter werden nachfolgend im Einzelnen erläutert.

\section{Einwohnerzahlen}

Als Einwohnerzahl pro Bezirk und für Berlin insgesamt wird die durchschnittliche Bevölkerung des Jahres 2010 gemäß Amt für Statistik Berlin-Brandenburg verwendet.

\section{Ärztezahlen}

Die Ärztezahlen werden dem Arztregister der KV Berlin mit Stand vom 1.1.2010 entnommen. Sie sind auf Vollzeitäquivalente umgerechnet, was gegenüber der üblichen Zählung von Arztsitzen den Vorteil größerer Genauigkeit hat, zumal die Anteile Vollzeit und Teilzeit arbeitender Ärztinnen und Ärzte in den verschiedenen Arztgruppen unterschiedlich ausfallen.

\section{Sozialindex I}

In die Beschreibung der Berliner Sozialstruktur gehen auf Bezirksebene 64 Einzelindikatoren aus den Bereichen Bevölkerung und Haushaltsstruktur, Bildung, Erwerbsleben, Einkommensquelle und Gesundheitszustand ein.

Um die komplexen Zusammenhänge der Vielzahl von Indikatoren auf wenige, interpretierbare Dimensionen zu reduzieren, wurde eine Faktorenanalyse der Einzelindikatoren auf der räumlichen Ebene der 12 Bezirke durchgeführt, die in drei Faktoren resultierte (vgl. Meinlschmidt 2009). Der Sozialindex I vereinigt nicht nur die meisten der eingehenden Einzelindikatoren auf sich, sondern weist auch mit $46 \%$ die höchste Varianzaufklärung der drei Faktoren auf und wird daher als Einzelindikator für die Sozialstruktur ausgewählt.

Der Sozialindex I bildet soziale Problemlagen im Bezirk wie beispielsweise eine erhöhte vorzeitige Sterblichkeit, eine hohe Abhängigkeit von Transfereinkommen und eine geringe berufliche Qualifikation ab. Aufgrund der Fakto- renanalyse sind die Werte des Sozialindex I z-standardisiert mit einem Mittelwert von 0 und einer Streuung von 1. Negative Werte bezeichnen eine problematische Sozialstruktur, positive Werte eine günstige Sozialstruktur im Bezirk.

\section{Morbiditätsindex I}

Zur Abbildung der Krankheitslast im Sozialraum wird ein Morbiditätsindex verwendet, der aus Abrechnungsdaten der KV Berlin des Jahres 2007 gewonnen wurde. Hierzu wurden für die 60 häufigsten Diagnosen sowie einige weitere sozialepidemiologisch bedeutsame Diagnosen wie Tuberkulose und Krebs verschiedener Lokalisationen altersstandardisierte Morbiditätsraten auf der Ebene der 447 Berliner Planungsräume berechnet. Über diese standardisierten Morbiditätsraten wurde eine Faktorenanalyse durchgeführt, die vier Faktoren zur Beschreibung der Morbidität im Raum ergab (vgl. Meinlschmidt 2011).

Auf dem ersten der vier Faktoren, der als Morbiditätsindex I bezeichnet wird, laden vor allem Infektionskrankheiten, psychische Störungen und psychosomatische Beschwerden, Adipositas, Krankheiten des Verdauungssystems und Verletzungen. Er klärt 32 \% der Gesamtvarianz der Morbiditätsraten auf. Damit eignet sich der Morbiditätsindex I sowohl für die psychotherapeutische als auch für die hausärztliche Versorgung als Indikator des Versorgungsbedarfs.

Für die Analysen zur ambulanten Versorgung wurde der Morbiditätsindex I von der Ebene der 447 Planungsräume auf die Ebene der 12 Bezirke bevölkerungsgewichtet hochaggregiert. Ebenso wie beim Sozialindex I sind auch die Werte des Morbiditätsindex I z-standardisiert mit einem Mittelwert von 0 und einer Streuung von 1. Negative Werte bedeuten eine überdurchschnittliche Krankheitsbelastung der Bevölkerung im Sozialraum, positive Werte eine unterdurchschnittliche Krankheitsbelastung.

\section{Wohnortnahe Versorgung}

In der Diskussion um die Bedarfsplanung spielt die wohnortnahe Versorgung eine große Rolle und wird daher auch in den vorliegenden Analysen berücksichtigt. Da es jedoch vielfältige Gründe für die Abweichung vom Prinzip 
der wohnortnahen Versorgung sowohl aus ärztlicher Perspektive als auch aus der Perspektive der Patient/innen gibt, geht dieser Aspekt zunächst nicht in die Modellrechnung ein.

Um die Wohnortnähe der Versorgung zu beschreiben, wird erfasst, zu welchen Anteilen die Ärztinnen und Ärzte Patient/innen versorgen, die im gleichen Bezirk wohnen, in dem die Arztpraxis liegt (bezirkliche Versorgung), oder Patien-

\section{Es gibt vielfältige Gründe, vom Prinzip der wohnortnahen Versorgung abzuweichen.}

\section{Modellbildung}

Zur Berechnung der Umverteilungseffekte, die aufträten, wenn die vorhandenen Arztsitze in Berlin auf Basis der Bevölkerungs-, Sozial- und Morbiditätsstruktur umverteilt würden, wird auf der Ebene der 12 Bezirke ein lineares Komponentenmodell spezifiziert. Das Modell basiert auf den aktuellen (Ist-)Arztzahlen für Psychotherapeuten und Hausärzte in Berlin. In einem ersten

tinnen und Patienten aus anderen Wohnbezirken (überbezirkliche Versorgung). Dabei werden gemäß dem Wohnortprinzip der Gesundheitsberichterstattung ausschließlich Ärzt/innen und Patient/ innen mit Wohnort in Berlin betrachtet, die quantitativ ebenfalls nicht unbedeutende Mitversorgung von Patient/innen aus dem Berliner Umland durch Berliner Ärzt/innen bleibt außer Betracht.

Statt der Patientenzahl wird die Zahl der Abrechnungsscheine zugrunde gelegt, weil hierdurch die mehrmalige oder regelmäßige Inanspruchnahme und somit der Versorgungsumfang besser abgebildet wird.

\section{Ärztliche Auslastung}

Die von den Ärztinnen und Ärzten im Bezirk erbrachte Versorgungsleistung lässt sich anhand der Zahl der behandelten Patienten bzw. der Abrechnungsscheine abbilden. Zur Berücksichtigung mehrmaliger Inanspruchnahme wird wiederum die Zahl der Abrechnungsscheine herangezogen.

Für die Psychotherapeuten und Hausärzte wird für jeden Bezirk die durchschnittliche Zahl der pro Arzt abgerechneten Scheine als Indikator für die ärztliche Auslastung berechnet. Da kein Anhaltspunkt dafür vorliegt, welche Zahl an Patienten bzw. Scheinen pro Arzt als angemessen zu betrachten ist und somit kein absoluter Maßstab für die Auslastung, Unter- oder Überlastung vorliegt, dienen diese Angaben lediglich dem Vergleich zwischen den Bezirken.

$\mathrm{Zu}$ beachten ist die bereits erwähnte unterschiedliche Versorgungsform von Hausärzten und Psychotherapeuten, die
Schritt erfolgt eine Aufteilung der Ärzt/ innen der jeweiligen Arztgruppe auf die Bezirke entsprechend ihrem Bevölkerungsanteil (bevölkerungsgewichtete Umverteilung). Hierdurch wird für alle Berliner Bezirke eine gleiche Bevölkerungs-Arzt-Relation hergestellt.

Im zweiten Schritt werden die so ermittelten bezirklichen Soll-Arztzahlen mit einem bezirksspezifischen Korrekturfaktor für die Sozialstruktur und einem bezirksspezifischen Korrekturfaktor für die Morbiditätsstruktur multipliziert, um das Ärztesoll unter Berücksichtigung von Sozialstruktur und Morbidität zu errechnen. Im Sinne eines additiven Modells wird dabei die Hälfte der Gesamtzahl der Ärzt/innen mit dem Korrekturfaktor für die Sozialstruktur gewichtet, die andere Hälfte mit dem Korrekturfaktor für die Morbiditätsstruktur. Damit wird beiden Korrekturfaktoren das gleiche Gewicht im Modell beigemessen.

Die Berechnungsformel für das Ärztesoll unter Berücksichtigung von Sozialstruktur und Morbidität (ASSIMI) lautet folgendermaßen:

$$
\begin{aligned}
\operatorname{ASSIMI}_{i}= & \frac{A}{2} * \frac{B_{i} *\left(S I_{i}(-1)+\left|\operatorname{Min}\left(S I_{i}(-1)\right)\right|+1\right)}{\sum_{i} B_{i} *\left(S I_{i}(-1)+\left|\operatorname{Min}\left(S I_{i}(-1)\right)\right|+1\right)}+ \\
& +\frac{A}{2} * \frac{B_{i} *\left(M I_{i}(-1)+\left|\operatorname{Min}\left(M I_{i}(-1)\right)\right|+1\right)}{\sum_{i} B_{i} *\left(M I_{i}(-1)+\left|\operatorname{Min}\left(M I_{i}(-1)\right)\right|+1\right)}
\end{aligned}
$$

$B_{i}$ : Bevölkerung des Bezirkes i

$A$ : Ärzte insgesamt

$S I$ : Sozialindex I des Bezirkes i

$M I_{i}$ : Morbiditätsindex I des Bezirkes i

Durch Abgleich der vorgefundenen Ärztezahlen pro Bezirk mit dem durch die Modellformel berechneten Ärztesoll wird die Zahl der gemäß Modell um- zuverteilenden Ärzte quantifiziert. Diese Abweichung von Ist- und Soll-Ärztezahl wird als »Fehlallokation« bezeichnet.

\section{Ergebnisse}

Die zwölf Berliner Bezirke weisen mit Einwohnerzahlen zwischen 220.000 und 370.000 jeweils eine Großstadtbevölkerung auf. Nach der Bedarfsplanungsrichtlinie ist Berlin eine einheitliche Planungsregion der Regionalklasse 1 mit einer als bedarfsgerecht betrachteten Allgemeinen Verhältniszahl von 1.585 für Hausärzte und 2.577 für Psychotherapeuten.

Angesichts der tatsächlichen Zahlen von zur ambulanten Versorgung zugelassenen Hausärzt/innen und Psychotherapeut/innen (Stand Januar 2010) errechnen sich für ganz Berlin Versorgungsgrade von $109 \%$ für die Hausärzte und $157 \%$ für die Psychotherapeuten. Allerdings verteilen sich die Hausärzt/innen und Psychotherapeut/innen ungleich über die Berliner Bezirke (vgl. Tabelle 1). Bezüglich der Hausärzt/innen berechnet sich für die Bezirke ein Versorgungsgrad zwischen $89 \%$ (Lichtenberg) und $144 \%$ (Charlottenburg-Wilmersdorf). Vier der zwölf Bezirke sind mit einem Versorgungsgrad von über $110 \%$ als überversorgt zu betrachten. Bei den Psychotherapeut/innen variiert der Versorgungsgrad weit stärker zwischen den Bezirken und reicht von $32 \%$ (Marzahn-Hellersdorf) bis zu $418 \%$ (Charlottenburg-Wilmersdorf). Sechs der zwölf Bezirke sind überversorgt, für Marzahn-Hellersdorf ist eine Unterversorgung festzustellen.

Auch die soziale und morbiditätsbedingte Belastung der Bevölkerung verteilen sich ungleich über die Stadtbezirke (vgl. Abbildungen 1 und 2). Am höchsten sozial belastet sind die Bezirke Mitte und Neukölln, gefolgt von FriedrichshainKreuzberg, die günstigste Sozialstruktur weisen die Bezirke Steglitz-Zehlendorf und Charlottenburg-Wilmersdorf auf, gefolgt von Treptow-Köpenick. Der Morbiditätsindex I (vgl. Abbildung 2) weist die höchste Krankheitsbelastung aus für die Bezirke Neukölln, Friedrichshain-Kreuzberg und Mitte, die zugleich auch die mit der höchsten sozialstrukturellen Belastung sind. Die geringste Krankheitsbelastung errechnet sich für die Bezirke Pankow, Steglitz-Zehlendorf und Treptow-Köpenick. 
Die aus Tabelle 1 ersichtliche Ähnlichkeit der Verteilung sozialer und morbiditätsbedingter Belastungen über die Bezirke drückt sich in einer signifikanten Korrelation von Sozialindex I und Morbiditätsindex I in Höhe von $\mathrm{r}=0,77$ aus. Allerdings ist die derzeitige Versorgungssituation nicht auf den Versorgungsbedarf zugeschnitten, der sich aus der sozialen und morbiditätsbedingten Belastung ablesen lässt. Die Versorgungsgrade korrelieren mit dem Sozialindex I auf Bezirksebene zwar nicht signifikant, aber positiv (Hausärzte $r=0,15$, Psychotherapeuten $r=0,49$ ). Das bedeutet, dass die Versorgung in den Bezirken mit günstiger Sozialstruktur besonders gut ist, ist Bezirken mit hoher sozialer Belastung dagegen unterdurchschnittlich. Mit dem Morbiditätsindex I weisen die Versorgungsgrade keinerlei Zusammenhang auf (Hausärzte $r=-0,06$, Psychotherapeuten $r=0,11$ ).

Die Auswertung zur wohnortnahen Versorgung zeigt, dass die Hausärzt/ innen zu etwa drei Vierteln Patient/innen im gleichen Bezirk versorgen und zu knapp einem Viertel Patient/innen, die in einem anderen Bezirk wohnen. In der Berufsgruppe der Psychotherapeut/ innen macht dagegen die bezirkliche Versorgung weniger als die Hälfte aus, die überbezirkliche Versorgung dagegen etwas mehr als die Hälfte. Auch bezüg- lich der wohnortnahen Versorgung bestehen deutliche bezirkliche Unterschiede (vgl. Tabelle 2). Am höchsten ist der Anteil bezirklicher Versorgung durch Hausärzt/innen in den eher am Stadtrand gelegenen Bezirken Pankow, Steglitz-Zehlendorf und Reinickendorf, am niedrigsten in den zentral gelegenen Bezirken Mitte, Friedrichshain-Kreuzberg und Charlottenburg-Wilmersdorf. Bezüglich der psychotherapeutischen Versorgung sind die bezirklichen Unterschiede noch erheblicher als bei den Hausärzten. In den am Stadtrand gelegenen Bezirken mit eher niedrigem Versorgungsgrad ist ein hoher Anteil bezirklicher Versorgung zu verzeichnen, so in Marzahn-Hellersdorf und Spandau. In den zentraleren Bezirken mit hohem Versorgungsgrad wie CharlottenburgWilmersdorf und Mitte ist der überbezirkliche Versorgungsanteil dagegen besonders hoch.

Die ärztliche Auslastung - gemessen an der Zahl der Abrechnungsscheine pro Arzt bzw. Therapeut im Jahr 2007 - kann nicht über die Arztgruppen verglichen werden, da sich die Versorgungsangebote von Hausärzten und Psychotherapeuten grundlegend unterscheiden. Die absolute Zahl der Abrechnungsscheine kann nicht dazu herangezogen werden, zu beurteilen, ob die Ärzt/innen bzw. Therapeut/innen "genug« arbeiten.
Sie soll lediglich dazu dienen, regionale Unterschiede in der Intensität der Inanspruchnahme abzubilden.

Aus Tabelle 2 ist ersichtlich, dass die ärztliche Auslastung zwischen den Bezirken bei den Hausärzten um den Faktor 1,5 schwankt, bei den Psychotherapeuten um den Faktor 1,8. Dabei ist die Auslastung in Bezirken mit hohem Versorgungsgrad eher niedrig und umgekehrt (Hausärzte $r=-0,76$, Psychotherapeuten $r=-0,62)$. Die Korrelationen der ärztlichen Auslastung mit der Sozial- und Morbiditätsstruktur sind nicht signifikant.

Durch das im Methodenteil spezifizierte Modell wird die in Berlin zum 1.1.2010 tatsächlich vorhandene Ärztezahl auf die zwölf Bezirke verteilt, indem das Ärztesoll unter Berücksichtigung von Einwohnerzahlen, Sozial- und Morbiditätsstruktur errechnet wird.

Die Zahlen in Tabelle 3 zeigen, dass die Berücksichtigung von Sozial- und Morbiditätsstruktur mehrheitlich in die gleiche Richtung wirken, was aufgrund des Zusammenhangs von sozialer und krankheitsbedingter Belastung der Bevölkerung in den Bezirken zu erwarten war.

Die Differenz von tatsächlichen Ärzte- bzw. Therapeutenzahlen im Bezirk (vgl. Tabelle 1) und den unter Berücksichtigung von Bevölkerungszahlen, So-

Tabelle 1: Bevölkerung, ärztliche Versorgung, Sozial- und Morbiditätsstruktur in den Berliner Bezirken

\begin{tabular}{|c|c|c|c|c|c|c|c|}
\hline \multirow{2}{*}{ Bezirk } & \multirow{2}{*}{ Bevölkerung } & \multicolumn{2}{|c|}{ Ärzte (Vollzeitäquivalente) } & \multicolumn{2}{|c|}{ Versorgungsgrad } & \multirow{2}{*}{$\begin{array}{l}\text { Sozial- } \\
\text { index I }\end{array}$} & \multirow{2}{*}{$\begin{array}{c}\text { Morbiditäts- } \\
\text { index I }\end{array}$} \\
\hline & & Hausärzte & Psychoth. & Hausärzte $^{1}$ & Psychoth. $^{2}$ & & \\
\hline Mitte & 332.689 & 246,85 & 193,70 & $118 \%$ & $150 \%$ & $-1,40$ & $-0,50$ \\
\hline Friedrichshain-Kreuzberg & 268.688 & 190,35 & 149,75 & $112 \%$ & $144 \%$ & $-1,22$ & $-0,82$ \\
\hline Pankow & 367.846 & 235,50 & 159,10 & $101 \%$ & $111 \%$ & 0,52 & 0,71 \\
\hline Charlottenburg-Wilmersdorf & 320.107 & 291,65 & 519,00 & $144 \%$ & $418 \%$ & 0,98 & 0,20 \\
\hline Spandau & 224.666 & 148,60 & 74,80 & $105 \%$ & $86 \%$ & $-0,32$ & $-0,33$ \\
\hline Steglitz-Zehlendorf & 294.564 & 199,40 & 303,20 & $107 \%$ & $265 \%$ & 1,79 & 0,52 \\
\hline Tempelhof-Schöneberg & 335.476 & 266,00 & 343,55 & $126 \%$ & $264 \%$ & 0,31 & $-0,44$ \\
\hline Neukölln & 310.780 & 185,10 & 85,40 & $94 \%$ & $71 \%$ & $-1,37$ & $-1,51$ \\
\hline Treptow-Köpenick & 241.798 & 139,75 & 83,00 & $92 \%$ & $88 \%$ & 0,92 & 0,49 \\
\hline Marzahn-Hellersdorf & 248.866 & 157,30 & 31,10 & $100 \%$ & $32 \%$ & $-0,39$ & 0,32 \\
\hline Lichtenberg & 260.490 & 146,50 & 61,10 & $89 \%$ & $60 \%$ & $-0,05$ & 0,31 \\
\hline Reinickendorf & 240.963 & 164,00 & 90,20 & $108 \%$ & $96 \%$ & 0,24 & $-0,08$ \\
\hline Berlin gesamt & 3.446 .974 & $2.371,00$ & $2.093,90$ & $109 \%$ & $157 \%$ & 0,00 & $-0,09$ \\
\hline
\end{tabular}

${ }^{1}$ Allgemeine Verhältniszahl (Einwohner pro Arzt) laut Bedarfsplanungsrichtlinie: 1.585

${ }^{2}$ Allgemeine Verhältniszahl (Einwohner pro Arzt) laut Bedarfsplanungsrichtlinie: 2.577

Quelle: Bevölkerung: Amt für Statistik Berlin-Brandenburg, Ärzte: KV Berlin, Versorgungsgrad, Sozial- und Morbiditätsindex: eigene Berechnungen Senatsverwaltung für Gesundheit und Soziales Berlin 
zial- und Morbiditätsindex berechneten Soll-Ärztezahlen soll hier als Fehlallokation interpretiert werden und ist in Abbildung 3 für die zwölf Bezirke grafisch aufbereitet. Dabei bedeuten positive Zahlen, dass im Bezirk zu viele Ärzte bzw. Therapeuten ansässig sind, negative Zahlen weisen auf zu wenige Arztsitze im Bezirk hin.

Aus der Modellrechnung ergibt sich für die Hausärzte in Berlin eine Fehlallokation von insgesamt 749 Arztsitzen, das sind fast ein Drittel aller Hausärzte in der Stadt. Die Bezirke, in denen die meisten Ärzte fehlen, sind zugleich die Bezirke mit der stärksten sozialen Belastung sind. Den größten Ärzteüberschuss haben die Bezirke zu verzeichnen, die auch eher eine positive Sozialstruktur aufweisen.

Eine Gegenüberstellung der Modellergebnisse für Hausärzte und Psychotherapeuten zeigt strukturell ähnliche Ergebnisse, allerdings fallen die Umverteilungseffekte aufgrund der Modellrechnung für Psychotherapeuten größer aus als für Hausärzte (vgl. Abbildung 3). Dies überrascht nicht angesichts der Tatsache, dass die Psychotherapeuten sich aktuell weit ungleichmäßiger über die Bezirke verteilen als die Hausärzte (vgl. Tabelle 1).

Allein ein Viertel aller Berliner Psychotherapeut/innen mit Kassenzulassung hat den Praxissitz im Bezirk Charlottenburg-Wilmersdorf, was zu einem entsprechend großen Psychotherapeutenüberschuss dieses Bezirks in der Modellrechnung führt. Ebenfalls hohe Überschüsse haben die Bezirke SteglitzZehlendorf und Tempelhof-Schöneberg aufzuweisen. Den größten Fehlbedarf an Psychotherapeuten weist die Modellrechnung für den Bezirk Neukölln aus, der von gleich fünf weiteren Bezirken mit ähnlich hoher negativer Fehlallokation von Psychotherapeuten gefolgt wird, darunter Marzahn-Hellersdorf als bezüglich dieser Arztgruppe rechnerisch unterversorgter Bezirk. Insgesamt müssten nach der Modellrechnung sieben von zehn Psychotherapeuten ihre Praxis in einen anderen Bezirk verlegen, um eine bedarfsgerechte Versorgung zu gewährleisten.

In den Abbildungen $3 \mathrm{~b}$ ) und d) ist die nach dem Modell berechnete Fehlallokation danach aufgeschlüsselt, welcher Anteil der ausgewiesenen Fehlallokation auf die Adjustierung nach der Bezirks-

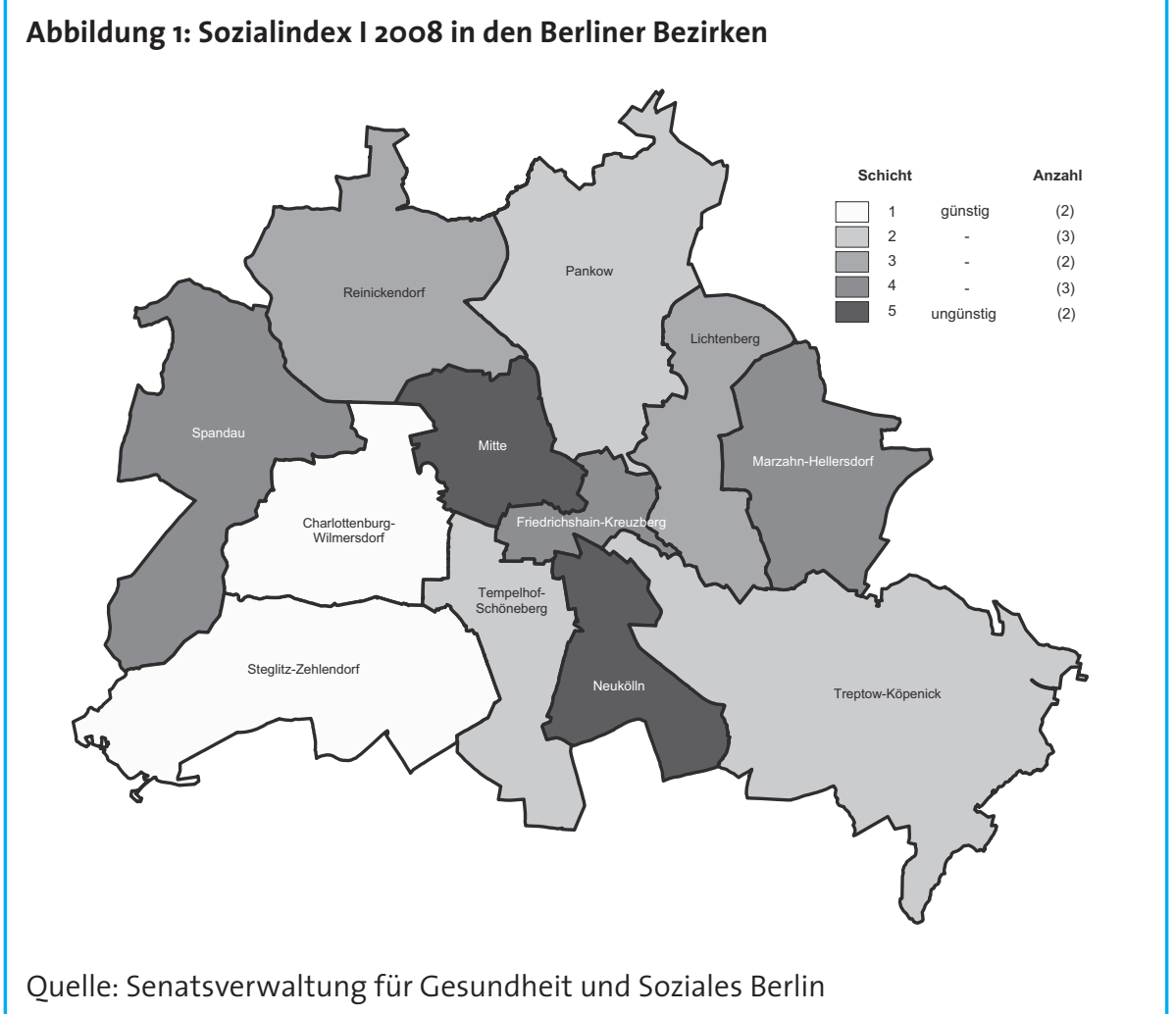

bevölkerung zurückgeht, welcher Anteil auf den Sozialindex I und welcher Anteil auf den Morbiditätsindex I. Mehrheitlich weisen die verschiedenen Modellparameter Umverteilungseffekte jeweils in die gleiche Richtung pro Bezirk aus, es gibt aber auch Bezirke, in denen sie in unterschiedliche Richtung weisen.
Weiterhin lässt sich in der Aufschlüsselung erkennen, dass CharlottenburgWilmersdorf gemäß der Modellrechnung vor allem deshalb Psychotherapeuten an andere Bezirke abgeben muss, zahl viel zu viele hat. Dagegen bekommt Neukölln vor allem aufgrund der unweil es gemessen an der Bevölkerungs-

\section{Abbildung 2: Morbiditätsindex I in den Berliner Bezirken}

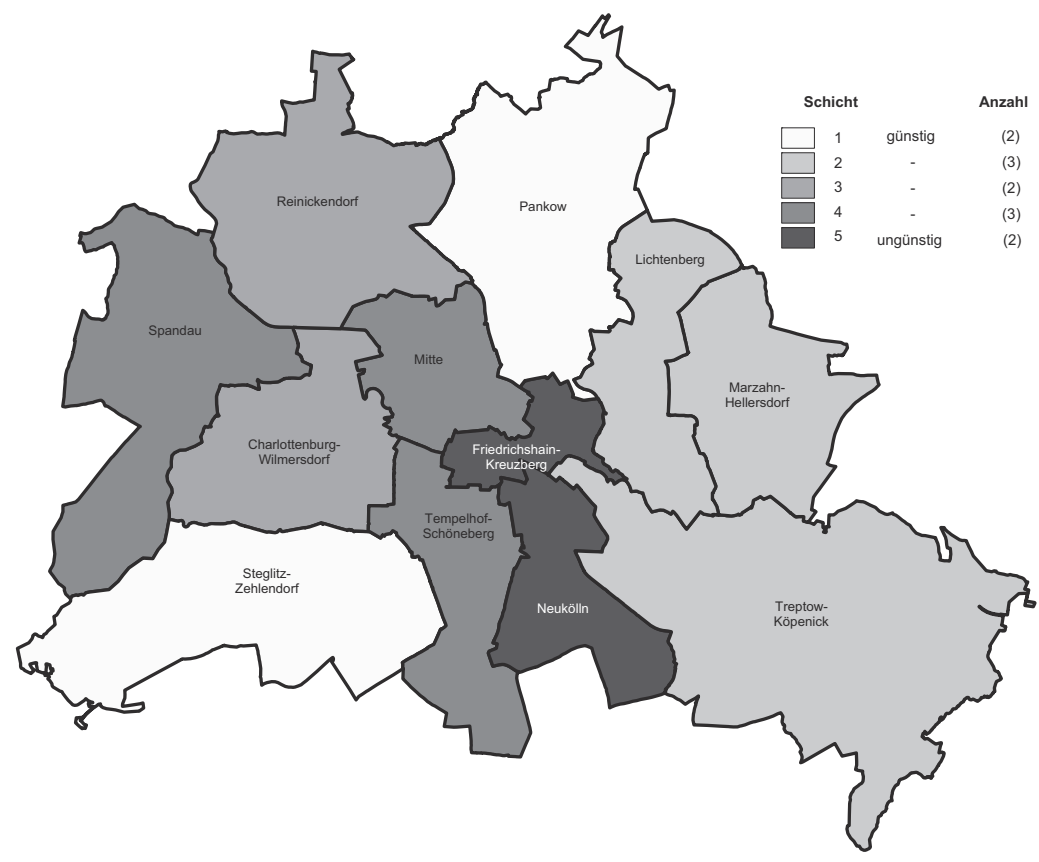

Quelle: Senatsverwaltung für Gesundheit und Soziales Berlin 


\section{Tabelle 2:}

Wohnortnahe Versorgung und ärztliche Auslastung in den Berliner Bezirken

\begin{tabular}{|c|c|c|c|c|c|c|}
\hline \multirow[b]{2}{*}{ Bezirk } & \multicolumn{3}{|c|}{ Hausärzte } & \multicolumn{3}{|c|}{ Psychotherapeuten } \\
\hline & $\begin{array}{c}\text { bezirkliche } \\
\text { Versorgung }^{1}\end{array}$ & $\begin{array}{l}\text { überbezirkliche } \\
\text { Versorgung }^{2}\end{array}$ & Auslastung $^{3}$ & $\begin{array}{c}\text { bezirkliche } \\
\text { Versorgung }^{1}\end{array}$ & $\begin{array}{l}\text { überbezirkliche } \\
\text { Versorgung }^{2}\end{array}$ & Auslastung $^{3}$ \\
\hline Mitte & $65,1 \%$ & $34,9 \%$ & 2.888 & $31,9 \%$ & $68,1 \%$ & 89 \\
\hline Friedrichshain-Kreuzberg & $60,0 \%$ & $40,0 \%$ & 3.011 & $43,3 \%$ & $56,7 \%$ & 94 \\
\hline Pankow & $81,6 \%$ & $18,4 \%$ & 3.199 & $63,6 \%$ & $36,4 \%$ & 119 \\
\hline Charlottenburg-Wilmersdorf & $61,1 \%$ & $38,9 \%$ & 2.356 & $31,7 \%$ & $68,3 \%$ & 80 \\
\hline Spandau & $90,3 \%$ & $9,7 \%$ & 3.003 & $80,0 \%$ & $20,0 \%$ & 93 \\
\hline Steglitz-Zehlendorf & $78,5 \%$ & $21,5 \%$ & 2.393 & $43,4 \%$ & $56,6 \%$ & 74 \\
\hline Tempelhof-Schöneberg & $65,6 \%$ & $34,4 \%$ & 2.843 & $33,9 \%$ & $66,1 \%$ & 72 \\
\hline Neukölln & $77,3 \%$ & $22,7 \%$ & 3.380 & $54,6 \%$ & $45,4 \%$ & 83 \\
\hline Treptow-Köpenick & $92,8 \%$ & $7,2 \%$ & 3.207 & $57,4 \%$ & $42,6 \%$ & 116 \\
\hline Marzahn-Hellersdorf & $89,4 \%$ & $10,6 \%$ & 3.515 & $81,8 \%$ & $18,2 \%$ & 131 \\
\hline Lichtenberg & $83,1 \%$ & $16,9 \%$ & 3.438 & $60,8 \%$ & $39,2 \%$ & 124 \\
\hline Reinickendorf & $86,0 \%$ & $14,0 \%$ & 2.963 & $74,7 \%$ & $25,3 \%$ & 79 \\
\hline Berlin gesamt & $76,5 \%$ & $23,5 \%$ & 2.969 & $46,0 \%$ & $54,0 \%$ & 87 \\
\hline
\end{tabular}

${ }^{1}$ Anteil der Patient/innen der Arztgruppe im Bezirk, die im selben Bezirk wohnen, in dem die Arztpraxis liegt, im Jahr 2007

${ }^{2}$ Anteil der Patient/innen der Arztgruppe im Bezirk, die in einem anderen Bezirk wohnen als die Arztpraxis liegt, im Jahr 2007

${ }^{3}$ durchschnittliche Anzahl der Abrechnungsscheine der Ärzt/innen der Arztgruppe im Bezirk im Jahr 2007

Quelle: eigene Berechnungen Senatsverwaltung für Gesundheit und Soziales Berlin

günstigen Morbiditätsstruktur deutlich mehr Hausärzte und Psychotherapeuten zugewiesen.

\section{Diskussion}

Die Bedarfsplanung für die vertragsärztliche Versorgung erfolgt bisher ausschließlich auf der Basis der Verhältniszahlen von Einwohner/innen und Ärzt/ innen. Bei einer Aufschlüsselung der vertragsärztlichen Versorgung auf die zwölf Bezirke Berlins zeigen sich bereits in der Einwohner-Arzt-Relation erhebliche Disparitäten. Diese werden verschärft, wenn zusätzlich Parameter des Versorgungsbedarfs wie die Sozialstruktur und die Morbiditätslast berücksichtigt werden. Aus den vorgestellten Auswertungen lässt sich schlussfolgern, dass die aktuelle vertragsärztliche Versorgungssituation in Berlin nicht nur die sozialstrukturell und morbiditätsbedingten Versorgungsbedarfe außer Acht lässt, sondern sich geradezu konträr zu diesen verhält: in Bezirken mit hoher sozialer Belastung und hoher Morbidität haben sich vergleichsweise weniger Ärztinnen und Ärzte niedergelassen als in Bezirken mit günstiger Sozialstruktur und geringer Morbidität.

Der Effekt der Niederlassungspräferenz für strukturell begünstigte Regi- onen wird auch bei Uhlemann \& Lehmann (2011) beschrieben und u. a. mit einem höheren Anteil an Privatpatient/ innen, besseren Aussichten auf einen späteren Praxisverkauf, eine geringere Belastung mit Notdiensten und eine attraktivere Infrastruktur begründet. Diese Einschätzung zeigt, dass eine sozialindikative Steuerung der ärztlichen Versorgung nicht möglich ist, ohne dass Anreizinstrumente für die Verlagerung von Praxissitzen in Gebiete mit höherem Versorgungsbedarf geschaffen und genutzt werden.

Das vorgestellte mathematische Modell zur Berechnung von Soll-Ärztezahlen auf der Ebene der Berliner Bezirke stellt dabei einen möglichen Ansatz dar und kann unter verschiedenen Aspekten zur Diskussion gestellt werden. Zunächst einmal ist zu bedenken, dass das berechnete Modell auf den Ist-Arztzahlen beruht. Ebenso wäre es denkbar, von den Soll-Arztzahlen gemäß der Bedarfsplanungsrichtlinie auszugehen. In diesem Fall ergäbe sich für beide betrachteten Arztgruppen berlinweit ein deutlicher Ärzteüberschuss. Angesichts des Fehlens von fachlich-inhaltlich begründeten absoluten Maßstäben für den Ärztebedarf wurde der relative Ansatz gewählt, da eine Reduktion der Ärztezahl noch unrealistischer erscheint als eine sozialindikative Umverteilung der vorhandenen Arztsitze.

Auch die Wahl der zwölf Berliner Bezirke als Analyseebene kann in Frage gestellt werden. Mit den zwölf Bezirken wurde eine räumliche Gliederung gewählt, die in den Jahren 2000 bis 2003 bereits in der Bedarfsplanung verwendet wurde, bevor Berlin eine einheitliche Planungsregion wurde. ${ }^{1}$ Dabei darf aber nicht übersehen werden, dass es auch innerhalb der zwölf Bezirke erhebliche sozialstrukturelle Disparitäten gibt, denen nur bei kleinräumigerer Betrachtung Rechnung getragen werden kann. Andererseits ist eine zu kleinräumige Sichtweise angesichts der begrenzten Zahl zu verteilender Arztsitze und der vergleichsweise guten Verkehrsinfrastruktur in Berlin nicht zu rechtfertigen. Soll ein sozialindikatives Planungsmodell auf die verschiedenen Arztgruppen angewendet werden, so erscheint eine jeweils auf die Größe und den Spezialisierungsgrad der Arztgruppe zugeschnittene räumliche Betrachtungsebene sinnvoll, wie sie auch im Reformmodell der KBV für die vertragsärztliche Bedarfs-

\footnotetext{
1 Vor 2000 wurden die 23 Berliner Bezirke (Altbezirke) als Planungsregionen verwendet, die im Zuge der Bezirksreform $2000 \mathrm{zu}$ den zwölf neuen Bezirken fusionierten.
} 
Tabelle 3:

Berechnung von Soll-Ärztezahlen auf Bezirksebene unter Berücksichtigung von Einwohnerzahl, Sozial- und Morbiditätsstruktur

\begin{tabular}{|c|c|c|c|c|c|c|c|c|}
\hline \multirow[b]{2}{*}{ Bezirk } & \multicolumn{4}{|c|}{ Soll Hausärzte $(n=2.371)$} & \multicolumn{4}{|c|}{ Soll Psychotherapeuten $(n=2.094)$} \\
\hline & $\begin{array}{c}\text { nach } \\
\text { Bevöl- } \\
\text { kerung }\end{array}$ & $\begin{array}{c}\text { nach Bev. } \\
\text { und } \\
\text { Sozialstruktur }\end{array}$ & $\begin{array}{l}\text { nach Bev. und } \\
\text { Morbiditäts- } \\
\text { struktur }\end{array}$ & Gesamt & $\begin{array}{c}\text { nach } \\
\text { Bevöl- } \\
\text { kerung }\end{array}$ & $\begin{array}{c}\text { nach Bev. } \\
\text { und } \\
\text { Sozialstruktur }\end{array}$ & $\begin{array}{l}\text { nach Bev. und } \\
\text { Morbiditäts- } \\
\text { struktur }\end{array}$ & Gesamt \\
\hline Mitte & 228,8 & 172,1 & 145,7 & 317,8 & 202,1 & 152,0 & 128,7 & 280,7 \\
\hline Friedrichshain-Kreuzberg & 184,8 & 133,2 & 138,3 & 271,5 & 163,2 & 117,6 & 122,2 & 239,8 \\
\hline Pankow & 253,0 & 102,9 & 56,5 & 159,4 & 223,5 & 90,9 & 49,9 & 140,8 \\
\hline Charlottenburg-Wilmersdorf & 220,2 & 71,6 & 87,4 & 159,0 & 194,5 & 63,2 & 77,2 & 140,4 \\
\hline Spandau & 154,5 & 86,4 & 89,6 & 175,9 & 136,5 & 76,3 & 79,1 & 155,4 \\
\hline Steglitz-Zehlendorf & 202,6 & 36,4 & 58,3 & 94,7 & 178,9 & 32,1 & 51,4 & 83,6 \\
\hline Tempelhof-Schöneberg & 230,8 & 102,6 & 142,6 & 245,3 & 203,8 & 90,6 & 126,0 & 216,6 \\
\hline Neukölln & 213,8 & 159,5 & 210,5 & 370,0 & 188,8 & 140,9 & 185,9 & 326,8 \\
\hline Treptow-Köpenick & 166,3 & 55,9 & 49,7 & 105,6 & 146,9 & 49,4 & 43,9 & 93,3 \\
\hline Marzahn-Hellersdorf & 171,2 & 97,8 & 61,0 & 158,7 & 151,2 & 86,3 & 53,8 & 140,2 \\
\hline Lichtenberg & 179,2 & 91,4 & 64,2 & 155,5 & 158,2 & 80,7 & 56,7 & 137,4 \\
\hline Reinickendorf & 165,7 & 75,8 & 81,8 & 157,6 & 146,4 & 66,9 & 72,2 & 139,2 \\
\hline Berlin gesamt & 2371,0 & 1185,5 & 1185,5 & 2371,0 & 2093,9 & 1047,0 & 1047,0 & 2093,9 \\
\hline
\end{tabular}

Quelle: eigene Berechnungen Senatsverwaltung für Gesundheit und Soziales Berlin

planung vorgesehen ist (Köhler 2012). So erscheint für hoch spezialisierte Arztgruppen wie Anästhesisten, Radiologen und fachärztlich tätige Internisten (mit verschiedenen Spezialisierungen) eine Planung auf der Bezirksebene nicht erforderlich und eine noch kleinräumigere Planung schon allein aufgrund der Ärztezahlen unsinnig.

Die mathematische Ausgestaltung des Modells entscheidet über die Richtung und Größe der ermittelten »Fehlallokation". Es kann sowohl über die Gewichtung der eingehenden Parameter diskutiert werden als auch darüber, ob die Gesamtzahl der Ärzt/innen der sozialindikativen Umverteilung unterzogen werden soll oder beispielsweise jedem Bezirk eine "Grundausstattung " an Ärzt/innen zuerkannt wird und nur die darüber hinaus gehenden Arztsitze sozialindikativ verteilt werden sollen.

Der im Modell verwendete Morbiditätsindex I eignet sich für die betrachteten Arztgruppen der Hausärzte und Psychotherapeuten, weil er Krankheiten einschließt, die das zentrale Behandlungsspektrum dieser beiden Berufsgruppen ausmachen. Für anders spezialisierte Arztgruppen wie beispielsweise Frauenärzte, Orthopäden oder Neurologen müssten passendere Indikatoren für den Versorgungsbedarf gefunden werden. Hierfür können durchaus Diagnosedaten verwendet werden, allerdings sollten für eine Indexbildung jeweils arztgruppenspezifische Diagnosen herangezogen werden. Die anhand der ausgewählten Diagnosen bestimmten Morbiditätsraten können unter Verwendung eines Gewichtungsfaktors, der den Versorgungsaufwand für die jeweilige Erkrankung abbildet, aufsummiert werden. Die Verteilung der gewichteten Morbiditätsindizes im Raum kann dann als Maß für den Versorgungsbedarf in Bezug auf die jeweilige Arztgruppe verwendet werden. Als Gewichtungsfaktoren sind die durch die ärztliche Behandlung verursachten diagnosespezifischen Kosten vorstellbar, beispielsweise in Form des abgerechneten Leistungsbedarfs (von Stillfried \& Czihal 2011). Alternativ könnten die arztgruppenspezifischen Diagnosen einer Faktorenanalyse unterzogen werden, um die Erklärungsgehalte der Einzeldiagnosen als Gewichtungsfaktoren zu verwenden.

Die Auswertung der vertragsärztlichen Inanspruchnahme auf der Ebene der Berliner Bezirke zeigt ein hohes Ausmaß überbezirklicher Versorgung, das auf den ersten Blick im Widerspruch zur Maxime wohnortnaher Versorgung zu stehen scheint. Über die Gründe für die Inanspruchnahme niedergelassener Ärzt/innen außerhalb des eigenen Wohnbezirks kann nur spekuliert werden. Eine Arztwahl nach Arbeitsort oder Erreichbarkeit statt nach Wohnortnähe, das Beibehalten vertrauter Ärzt/innen bei Umzug innerhalb der Stadt oder eine Präferenz für eine/n persönlich empfohlenen Ärzt/in kann eine erwünschte überbezirkliche Versorgung begründen. Aus ärztlicher Sicht mag eine überbe- zirkliche Versorgung wünschenswert sein, wenn - beispielsweise aufgrund hoher Arztdichte - eine Auslastung der Praxis allein mit bezirklichen Patient/innen nicht erreichbar ist. Die Ergebnisse zum Zusammenhang der Arztdichte im Bezirk mit der Zahl der Abrechnungsscheine pro Arzt deuten darauf hin, dass diese Konstellation eine Rolle spielt. Zugleich kann ein hohes Ausmaß überbezirklicher Versorgung unerwünscht sein, wenn es z. B. dadurch zu Stande kommt, dass Patient/innen aufgrund von Überlastung wohnortnaher Arztpraxen und der damit verbundenen Wartezeitproblematik sich gezwungen sehen, auf weiter entfernt liegende Praxen auszuweichen.

Zusätzlich ist $\mathrm{zu}$ berücksichtigen, dass Berliner Vertragsärzt/innen in nicht geringem Umfang Mitversorgungseffekte für das Berliner Umland erbringen (laut KV Berlin ca. $10 \%$, vgl. Schlitt 2011 bzw. laut ZI 8,2 \% Mitversorgung für andere Regionen durch Berlin, vgl. Czihal et al. 2012). Einer Mitversorgung der Umlandbevölkerung durch Ärzt/ innen in städtischen Ballungsgebieten wird in der Bedarfsplanungsrichtlinie zwar indirekt Rechnung getragen, indem für die meisten Arztgruppen die Einwohner-Arzt-Relation in den Regionalklassen 1 (hierzu gehört auch Berlin) und 5 (Kernstädte) günstiger ist als in den übrigen, eher ländlich geprägten Regionalklassen. Eine direkte Berücksichtigung finden diese Effekte jedoch nicht, und der zu erwartende Umfang der Mitversorgung von Patient/innen 


\section{Abbildung 3:}

Fehlallokation von Arztsitzen: Differenz von tatsächlichen Arztzahlen im Bezirk und Soll-Ärztezahlen unter Berücksichtigung von Bevölkerung,

Sozialstruktur und Morbidität

a) Hausärzte, Fehlallokation insgesamt; b) Hausärzte, Fehlallokation aufgeschlüsselt;

c) Psychotherapeuten, Fehlallokation insgesamt; d) Psychotherapeuten, Fehlallokation aufgeschlüsselt

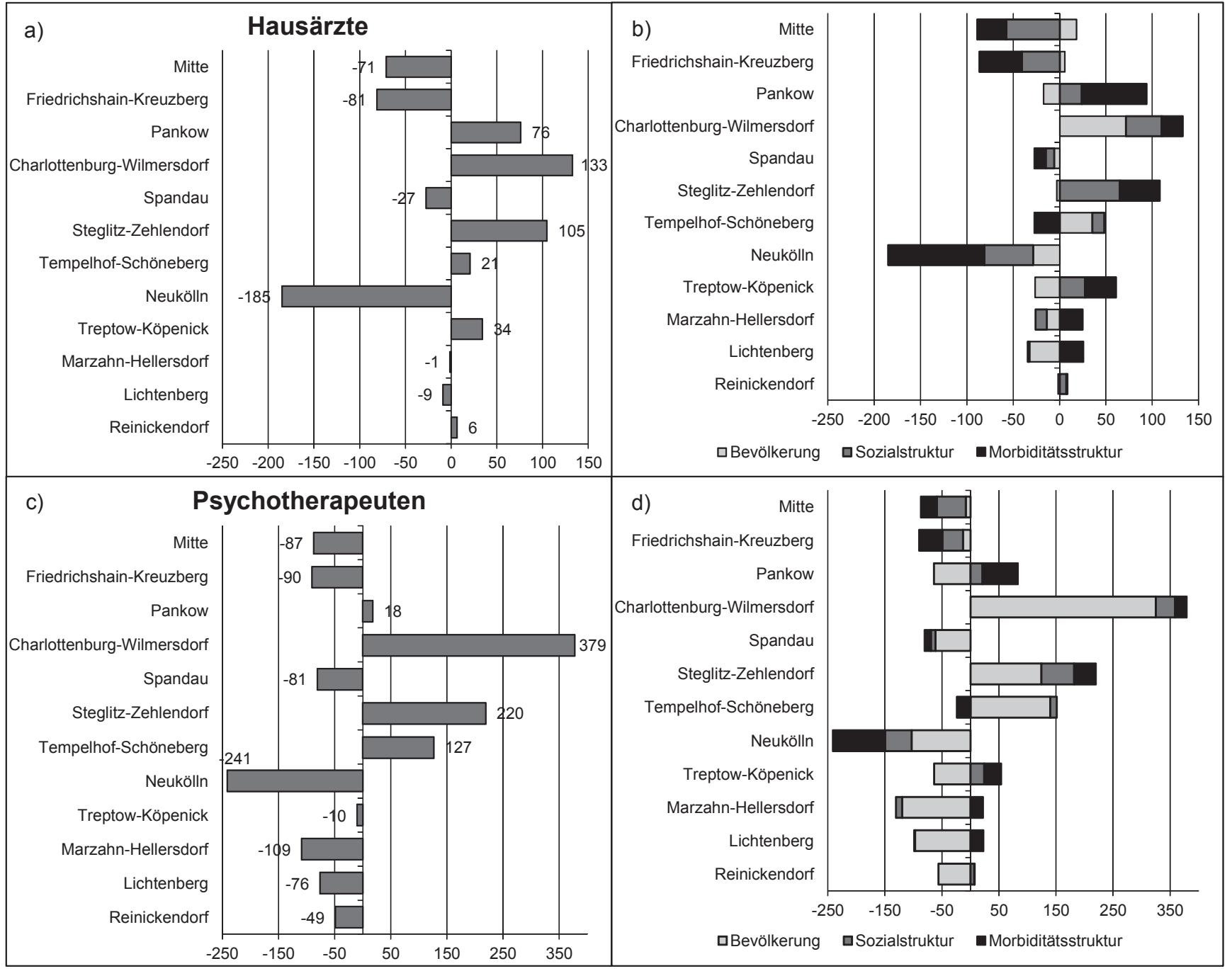

Quelle: Senatsverwaltung für Gesundheit und Soziales Berlin

aus anderen Regionen wird nicht beziffert.

Nicht zuletzt sind gerade im städtischen Raum Versorgungsstrukturen auch sektorübergreifend zu betrachten, da sowohl ein Erbringen von stationären Leistungen für Erkrankungen, die auch ambulant behandelt werden könnten

\section{Gerade im städtischen Raum sind Versorgungsstrukturen auch sektorübergreifend zu betrachten.}

Fachärzt/innen von Patient/innen, die in anderen Regionen, in denen diese spezialisierte Versorgung nicht zur Verfügung steht, stationär betreut werden müssten.

Neben den im Modell berücksichtigten Parametern zur Sozialstruktur und Morbidität ist eine Vielzahl weiterer Parameter denkbar, an denen die ärztliche Bedarfsplanung auszurichten ist, darunter die hier erwähnten Parameter zur Bevölkerungsstruktur, zur Inanspruchnahme und zur Versorgungsstruktur. Al(ambulant sensitive Krankenhausfälle), $\mathrm{zu}$ berücksichtigen ist als auch eine ambulante Behandlung durch spezialisierte lerdings sollte ein sozialindikatives Planungsmodell zur ambulanten ärztlichen Versorgung auch nicht mit zu vielen
Parametern und Gewichtungsfaktoren überlastet werden, damit es überschaubar, transparent und handhabbar bleibt.

\section{Politische Zielorientierung}

Das Versorgungsstrukturgesetz eröffnet neue Perspektiven und Gestaltungsmöglichkeiten sowohl für die regionale Bedarfsplanung als auch für die stärkere Einbeziehung der Länder in die ambulante und sektorübergreifende Versorgungsplanung und -steuerung. Mit dem hier vorgestellten Modell zur sozialindikativen Planung der regionalen ärztlichen Versorgung wird ein Diskussionsbeitrag für die Weiterentwicklung der ärztlichen Bedarfsplanung geleistet.

Die Senatsverwaltung für Gesundheit 
und Soziales Berlin setzt sich ein für die Etablierung eines gemeinsamen Landesgremiums gemäß $\$ 90$ a SGB V als einer Struktur zur institutionsübergreifenden Zusammenarbeit mit dem Ziel der Optimierung der medizinischen Versorgung in der Stadt. Die Rolle des Landes in Bezug auf dieses Gremium erschöpft sich nicht in der administrativen Funktion der Geschäftsführung, sondern soll darüber hinaus im Einbringen von Ideen und Vorschlägen sowie der methodischen Beratung und der Durchführung und Präsentation wissenschaftlicher Analysen zur Versorgungssituation bestehen.

Damit das Landesgremium gemeinsam mit dem Landesausschuss nach \$ 90 SGB V auch tatsächlich eine Steuerungswirkung entfalten kann, ist eine partnerschaftliche Zusammenarbeit der beteiligten Akteure erforderlich. Als Zielvorstellung wird eine Form der Kooperation gesehen, in der die beteiligten Institutionen jeweils ihre eigenen methodischen Kompetenzen, Erfahrungen, Vorstellungen und Datenbestände in den gemeinsamen politisch-wissenschaftlichen Diskurs einbringen, um im gemeinsamen Interesse aller eine bedarfsgerechte medizinische Versorgung für die Zukunft zu sichern.

\section{Die Auswirkungen des demographischen Wandels werden durch den Trend zur Spezialisierung verstärkt.}

Im Einzelnen ist Einvernehmen herzustellen über die Parameter, die in der Bedarfsplanung zu berücksichtigen sind, über die Bedeutung und Berücksichtigung von Patientenströmen über die Bezirksgrenzen hinweg und Mitversorgungsleistungen, die in erster Linie für Patient/innen aus dem Umland erbracht werden. Auch eine bessere Verzahnung von ambulanter und stationärer Versorgung steht auf der Agenda. Die strikte Trennung zwischen ambulanten und stationären Bereich gilt es, im Sinne einer patientenorientierten Bedarfsplanung zu überdenken. Eine stärkere fach- und sektor-übergreifende Zusam- menarbeit stellt für Patienten qualitativ hochwertige und gut erreichbare Facharztangebote sicher. Gleichzeitig bietet sie ökonomische Anreize, da teure technische Doppelstrukturen auf diese Weise vermieden werden könnten.

Im Hinblick auf die Erweiterung der Bedarfsplanung um demografische, sozialstrukturelle und morbiditätsbasierte Parameter ist es denkbar, Vorschläge und Modelle nicht nur auf der Landes-, sondern auch auf der Bundesebene in den Diskussionsprozess beispielsweise im Gemeinsamen Bundesausschuss der Ärzte und Krankenkassen einzubringen.

Im Rahmen der dort stattfindenden Überarbeitung der Bedarfsplanungsrichtlinie wird ebenfalls darüber $\mathrm{zu}$ diskutieren sein, im Sinne einer patientenorientierten Versorgung auch innerhalb von Facharztgruppen die Verhältniszahlen und die Größe der jeweiligen Planungsregion zu differenzieren. So hängen die Ansprüche an die Versorgungsstruktur bei Internisten eng mit der jeweiligen Schwerpunktbezeichnung zusammen. Aufgrund der notwendigen Frequenz der Arztbesuche wird daher für Internisten mit dem Schwerpunkt Diabetologie eine andere Versorgungsdichte als für Internisten mit dem Schwerpunkt Gastroenterologen benötigt. Aus diesen Überlegungen heraus empfiehlt sich eine Diskussion, ob nicht nur die Anzahl der von der Bedarfsplanung erfassten Arztgruppen erhöht, sondern auch eine Differenzierung innerhalb der Facharztgruppen vorgenommen werden sollte.

Letztendlich werden allerdings alle der im Rahmen der Bedarfsplanung diskutierten Modelle in Zukunft auch von der Problematik eines Mangels an Ärzten determiniert sein. Die Auswirkungen des demographischen Wandels werden durch den Trend zur Spezialisierung insbesondere im Bereich der Hausärzte verstärkt und es wird zu einer Herausforderung, freie Arztsitze zu vergeben. Diese Entwicklung unterstreicht die Notwendigkeit einer stärker als bisher an den spezifischen vor Ort existierenden Bedarfslagen ausgerichteten, fach- und sektorübergeifenden Planung.

\section{Literatur}

Czihal, T., von Stillfried, D. \& Schallock, M. (2012): Regionale Mitversorgungsbeziehungen in der ambulanten Versorgung. Berlin: ZI (Zentralinstitut für die kassenärztliche Versorgung in Deutschland). http://www. versorgungsatlas.de/themen/inanspruchnahme-der-versorgung, zuletzt aufgerufen 17.04.2012.

Klose, J. \& Rehbein, I. (2011): Ärzteatlas 2011. Daten zur Versorgungsdichte von Vertragsärzten. Berlin: Wissenschaftliches Institut der AOK (WidO).

Köhler, A. (2012): Neuausrichtung der Bedarfsplanung. Für eine gute Zukunft der ambulanten ärztlichen Versorgung. Deutsches Ärzteblatt, 109(1/2), A8-A10.

Kopetsch, T. (2011): Bedarfsplanung - Das Reformkonzept der Kassenärztlichen Bundesvereinigung. Gesundheits- und Sozialpolitik, 65, 1/2011, 34-42.

Meinlschmidt, G. (Hrsg.) (2009): Sozialstrukturatlas 2008 - Ein Instrument der quantitativen, interregionalen und intertemporalen Sozialraumanalyse. Berlin: Senatsverwaltung für Gesundheit, Umwelt und Verbraucherschutz.

Meinlschmidt, G. (Hrsg.) (2011): Basisbericht 2010/2011. Gesundheitsberichterstattung Berlin - Daten des Gesundheits- und Sozialwesens. Berlin: Senatsverwaltung für Gesundheit, Umwelt und Verbraucherschutz.

Mühlbacher, A.C. \& Wessels, M. (2011): Neue Wege in der Bedarfsplanung in Deutschland: Haben wir ein Mengen- oder ein Verteilungs- bzw. Allokationsproblem? Gesundheits- und Sozialpolitik, 65, 1/2011, $53-63$.

Schlitt, R. (2011): Die Illusion der kleinräumigen Bedarfsplanung. Eine schöne neue Welt, in der am Ende jedoch nichts besser würde? KV-Blatt 05/2011, 22-23.

Uhlemann, T. \& Lehmann, K. (2011): Reformkonzept des GKV-Spitzenverbandes zur Weiterentwicklung der Bedarfsplanung. Gesundheits- und Sozialpolitik, 65, 1/2011, 26-33.

von Stillfried, D. \& Czihal, T. (2011): Möglichkeiten der fachgruppenspezifischen Risikoadjustierung der Verhältniszahlen für eine zeitgemäße Versorgungsplanung. Gesundheits- und Sozialpolitik, 65, 2/2011, 29-36. 